\title{
NOUVEAUX SCHISTOSOMES D'OISEAUX DU GENRE GIGANTOBILHARZIA ODHNER
}

\author{
Par Alex FAIN
}

Nous avons publié récemment les résultats de nos observations sur les Schistosomes d'Oiseaux au Ruanda-Urundi. Dans ce matériel figuraient notamment deux nouvelles espèces du genre Gigantobilharzia Odhner (voir Fain, $1955 b$ et $1955 c$ ). L'examen de nouveaux hôtes dans ces mêmes régions nous a permis de découvrir encore trois nouvelles espèces appartenant à ce genre. Nous les décrirons ici et nous terminerons cette note en donnant une clé du genre Gigantobilharzia, basée sur les mâles de toutes les espèces connues jusqu'ici.

Le genre Gigantobilharzia constitue actuellement, si on y ajoute nos trois nouvelles espèces, un petit groupe de 13 espèces qui, à première vue, paraissent assez disparates. On y rencontre en effet des espèces qui présentent un canal gynécophore plus ou moins bien formé (11 espèces), à côté d'autres complètement démunies de ce canal $(G$. monocotylea et $G$. egreta).

Le manque d'homogénéité de ce groupe apparait aussi dans un autre caractère, celui de la ventouse buccale. Cette ventouse fait défaut chez toutes les espèces, sauf chez G. gyrauli (où elle existe dans les deux sexes), G. monocotylea (où elle existe seulement chez le mâle) et $G$. tantali (où elle existe à l'état rudimentaire dans les deux sexes).

Nous ne pensons pas que la présence d'une ventouse buccale chez certaines espèces constitue un caractère suffisant pour les exclure du genre Gigantobilharzia, d'autant plus que ce caractère semble être en pleine évolution dans ce genre.

L'absence complète de canal gynécophore revêt à nos yeux une importance systématique beaucoup plus grande, même si on tient compte qu'il existe chez plusieurs espèces de ce genre une certaine tendance à l'atrophie de cet organe. A notre avis, il n'est pas justifié de maintenir dans le genre Gigantobilharzia des espèces complètement dépourvues de canal gynécophore, comme cela sem- 
ble être le cas pour G. monocotylea Szidat et G. egreta Lal. La place de celles-ci ne serait done plus dans le genre Gigantobilharzia, mais bien dans un genre nouveau. Nous hésitons toutefois à prendre une telle décision pour le moment, car il existe dans notre esprit un doute quant à l'absence réelle de ce canal chez ces deux espèces. Il nous parait donc plus prudent d'attendre qu'un nouvel examen de ces espèces apporte éventuellement la confirmation de ce caractère et nous fixe sur la conduite à tenir. En attendant, nous maintiendrons provisoirement ces deux espèces dans le genre Gigantobilharzia.

Avant de décrire notre nouveau matériel, nous voudrions faire quelques remarques sur certains caractères morphologiques du genre Gigantobilharzia. La cuticule chez les cinq espèces étudiées par nous est dépourvue d'épines ou de petites élevures quelconques. Le canal gynécophore présente un développement très variable; il est très peu marqué et très court chez $G$. plectropteri, alors que, chez d'autres espèces, il est beaucoup mieux formé et peut atteindre une longueur appréciable. Notons que, chez G. adami, le canal gynécophore proprement dit est prolongé vers l'arrière par une gouttière assez superficielle, quoique bien distincte, creusée sur la face ventrale de la région testiculaire. Cette gouttière va jusqu'à l'extrémité postérieure du corps. Contrairement à ce que l'on observe dans le genre Trichobilharzia, où les parois et souvent le plancher du canal sont couverts de petites épines, chez les espèces du genre Gigantobilharzia, le canal gynécophore n'est généralement pas épineux, à l'exception toutefois d'une petite zone peu étendue en arrière de la papille génitale et qui peut être couverte de petites épines chez certaines espèces. Chez quatre des cinq espèces que nous avons examinées, les parois et le fond du canal gynécophore sont renforcés par une structure cuticulaire en forme d'échelle. Le nombre d'échelons constituant cette échelle semble constant dans une espèce donnée et ce caractère peut donc servir pour séparer les espèces entre elles. Chez G. tantali, cette structure scalariforme est finement épineuse. Le cirre, de même que la papille mâle sont généralement épineux dans le genre Gigantobilharzia. Nous avons noté (Fain, 1956) la présence dans le genre Trichobilharzia de deux vésicules séminales. Nous observons la même disposition chez toutes les espèces du genre Gigantobilharzia que nous avons examinées. La vésicule antérieure est libre dans le parenchyme, alors que la vésicule postérieure est complètement incluse à l'intérieur de la poche du cirre. Signalons que, chez $G$. plectropteri, les vésicules étaient très peu visibles à cause d'une certaine macération des spécimens, ce qui ne nous a pas permis d'en tracer les limites exactes. 


\section{GIGANTOBILHARZIA ADAMI n. sp. *.}

Cette nouvelle espèce est représentée dans notre collection par un mâle complet et deux fragments antérieurs de mâles. Ces spécimens furent récoltés dans les veines mésentériques du Canard nain.

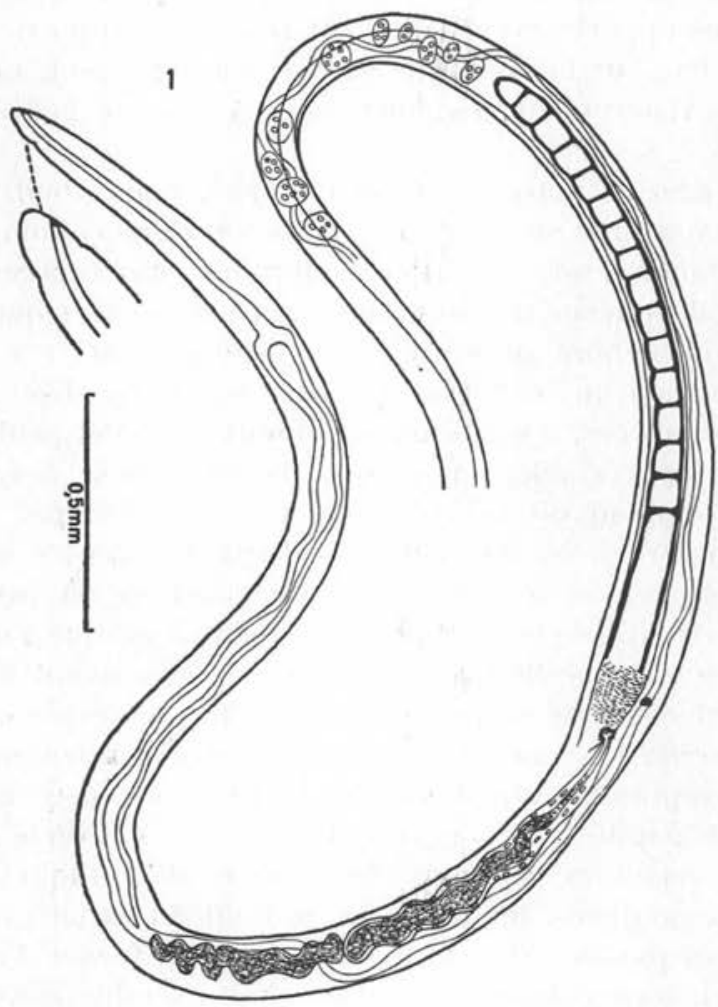

Fıg. 1. - Gigantobilharzia adami n. sp. : Mâle, partie antérieure avec début de la zone testiculaire.

Mâle (holotype) (fig. 1) : Mesuré en formol avant coloration et montage, le type mesure $21 \mathrm{~mm}$. de long. Après montage en baume, il mesure encore $20,13 \mathrm{~mm}$. de long. Les deux fragments de mâle sont longs respectivement de $13 \mathrm{~mm}$. et $15 \mathrm{~mm}$.; ils comprennent toute la partie antérieure du ver, ainsi qu'une grande partie de la région testiculaire. Ces trois spécimens ont été examinés d'abord

(*) Cette espèce est dédiée amicalement au Professeur W. Adam, Conservateur à l'Institut Royal des Sciences Naturelles de Bruxelles. 
à frais avant toute coloration. Ils furent ensuite colorés au carmin et montés au baume. Nous n'avons pas observé de différences sensibles dans les dimensions avant ou après montage. Les dimensions que nous donnerons ici sont celles prises sur les spécimens montés au baume, sauf indication contraire. Le ver est subcylindrique et très légèrement aplati dorso-ventralement; sa largeur est d'environ $100 \mu$ au niveau de la bifurcation œsophagienne (95 et $110 \mu$ chez les paratypes). Il s'élargit en arrière, atteignant au niveau de la vésicule séminale une largeur de 110 à $125 \mu$ chez le type (130 $\mu$ chez un paratype) et 125 à $150 \mu$ au niveau du canal gynécophore. En arrière du canal gynécophore, le corps présente une largeur allant de 90 à $140 \mu$ chez le type. Extrémité postérieure du corps non spatulée, mais arrondie. La cuticule du ver est lisse. Il n'y a pas de ventouses buccale, ni ventrale. L'œsophage est long de $755 \mu$ (paratypes : 730 à $780 \mu$ ). Les deux cæca se réunissent à environ $1.600 \mu$ en arrière de la bifurcation de l'œsophage, soit immédiatement en avant de l'extrémité antérieure de la vésicule séminale externe. Chez le paratype $\mathrm{n}^{\circ} 2$, cependant, l'union cæcale se situe dans la première partie du canal gynécophore, donc beaucoup plus en arrière. Le cæcum unique est sinueux et passe entre les testicules. Il se termine en cul-de-sac au niveau de l'extrémité postérieure du ver. Canal gynécophore long de 1.400 à $1.500 \mu$, large en vue latérale de 125 à $150 \mu$. Sa première partie, longue de $500 \mu$ environ, est plus fortement renflée $(150 \mu)$ et plus profonde que sa partie postérieure (largeur : 120 à $130 \mu$ ). Le plancher de cette dernière partie est renforcé par une série d'étroites bandelettes cuticulaires transversales, non épineuses, reliant les faces internes du canal. Ces bandelettes sont régulièrement espacées et leur nombre est de 14 à 15 . La première bandelette est située à $480 \mu$ de la papille génitale, la dernière est visible immédiatement en avant du premier testicule. Nous avons observé précédemment des formations similaires chez deux nouvelles espèces de Gigantobilharzia que nous avons décrites du Ruanda-Urundi. Ces bandelettes disparaissent au niveau de la région testiculaire, mais les lèvres du canal gynécophore restent visibles, bien que très atténuées sur toute la longueur du ver. Le cirre garni d'épines fait saillie par l'orifice génital. Des petites épines longues de 5 à $8 \mu$ sont également visibles dans une petite zone s'étendant seulement jusqu'à environ $100 \mu$ en arrière de la papille génitale. Tout le reste du canal gynécophore est dépourvu d'épines. Testicules au nombre de 280, disposés sur un seul rang; les plus petits présentent un diamètre de $30 \mu$ environ, les plus grands sont ovalaires et mesurent jusqu'à $90 \times 60 \mu$ (moyenne : $75 \times 55 \mu$ ). Les derniers testicules s'arrêtent à une dis- 
tance d'environ 600 à $700 \mu$ de l'extrémité postérieure. Vésicule séminale externe longue de $420 \mu$, décrivant environ 13 boucles et se rétrécissant assez brusquement vers son tiers antérieur (diamètre minimum : $27 \mu$, maximum : $51 \mu$ ) ; vésicule séminale interne, contenue dans la poche du cirre, longue de $500 \mu$, large de 35 à $60 \mu$ et présentant environ 12 boucles. La distance entre la vésicule séminale interne et la papille génitale est de $170 \mu$ environ $(140 \mu$ chez un paratype).

Position systématique : Deux espèces de Gigantobilharzia ont été décrites d'Afrique : G. ardeolæ et $G$. tantali. La nouvelle espèce que nous décrivons ici est bien distincte de la première par la taille plus petite; le nombre moins élevé des testicules; la longueur plus grande et la structure différente du canal gynécophore, dont le plancher comporte des petits ponts cuticulaires beaucoup plus nombreux ; la structure différente des vésicules séminales, etc... Elle se différencie de $G$. tantali par l'absence complète de ventouse buccale ; la longueur plus petite du canal gynécophore proprement dit ; le nombre moins élevé des bandelettes cuticulaires transversales qui renforcent le plancher du canal gynécophore et l'absence complète de petites épines sur ces bandelettes, comme sur les faces latérales de ce canal ; les dimensions différentes des vésicules séminales, plus courtes, mais plus larges, etc... Cette nouvelle espèce est d'autre part bien distincte des cinq espèces munies d'un canal gynécophore décrites hors d'Afrique, par différents caractères, et notamment la longueur du corps, et du canal gynécophore, la structure de ce dernier, etc... (voir clé).

Hôte: Veines mésentériques du Canard nain, Nettapus auritus Bodd.

Localité et date: Astrida, 3 janvier 1956 (Ruanda-Urundi).

Types: Au Musée du Congo à Tervuren (*).

\section{GIGANTOBILHARZIA NETTAPI n. sp.}

Le seul exemplaire que nous possédons de cette espèce est un mâle complet. Nous l'avons découvert également chez le Canard nain, où il était associé à G. adami n. sp.

Cette nouvelle espèce est proche de G. adami, mais elle est plus courte et plus large et présente une structure différente du canal gynécophore et un nombre beaucoup plus petit de testicules.

(*) Les types de G. tantali et G. ardcola ainsi que ceux des nouvelles espèces de Trichobilharzia que nous avons décrites du Congo ont également été déposés par nous dans les collections de ce Musée. 
Mâle (holotype) (fig. 2-4) : Il est long de $15 \mathrm{~mm}$. mesuré en formol, ou $14 \mathrm{~mm}$. coloré et monté en baume. Cuticule lisse ou plus ou moins striée transversalement par endroits. Forme générale comme dans l'espèce précédente. Il y a peu de différences dans les mensurations faites avant ou après montage au baume. Les dimensions que nous donnons sont celles faites sur le spécimen coloré et

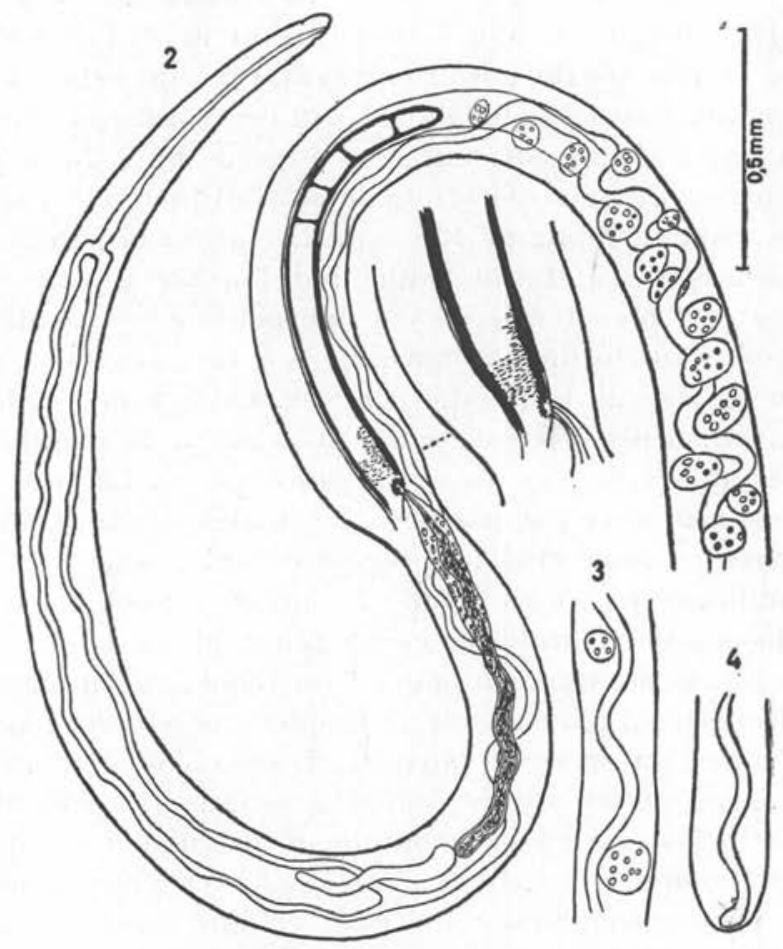

Fig. 2-4. - Gigantobilharzia nettapi n. sp. : Mâle, partie antérieure avec canal gynécophore et début de la zone testiculaire (2) ; milieu de la zone testiculaire (3) ; extrémité postérieure (4).

monté au baume. Largeur au niveau de la bifurcation de l'œsophage : $138 \mu$, de la vésicule séminale : $180 \mu$, du canal gynécophore : $210 \mu$ (en vue latérale), de la zone testiculaire : 150 à $200 \mu$. Il n'y a pas de ventouse buccale. La bouche s'ouvre ventralement, à environ $15 \mu$ de l'extrémité antérieure. L'œsophage est long de $725 \mu$. Les deux cæca s'unissent à environ $1.900 \mu$ en arrière de la bifurcation de l'œsophage. Le cæcum unique est sinueux et passe 
entre les testicules. Il aboutit au niveau de l'extrémité postérieure du corps. Le canal gynécophore est distant de $3.700 \mu$ de l'extrémité antérieure. La vésicule séminale externe (ou antérieure) est très transparente et peu visible, même après coloration. Elle est longue de $360 \mu$, de calibre inégal (25 à $45 \mu$ de diamètre) et décrit de 6 à 8 boucles. La vésicule séminale interne (ou postérieure), longue de $735 \mu$, présente une partie antérieure de calibre uniforme $(30-40 \mu$ de diamètre), longue de $450 \mu$ et décrivant de 6 à 7 boucles bien marquées, et une partie postérieure entourée de cellules prostatiques très nombreuses, longue de $285 \mu$ et décrivant également de 5 à 6 boucles ; sa moitié postérieure est large de 60 à $75 \mu$ (poche du cirre comprise) La vésicule séminale se continue vers l'arrière par le canal éjaculateur, long de $120 \mu$, qui aboutit à la papille génitale. Le cirre est épineux et fait saillie par l'orifice génital. Le canal gynécophore est bien formé. Il y a une petite zone garnie d'épines plus ou moins fusiformes (longues de 6 à $10 \mu$ environ), immédiatement en arrière de la papille génitale. Cette zone est longue de $100 \mu$ environ. Il n'y a pas d'épines en avant de la papille génitale, ni dans les autres parties du canal gynécophore. La face profonde du canal est renforcée par une série de petites bandes transversales non épineuses, comme chez l'espèce précédente, mais les ponts sont moins nombreux ici (5 au total). Le premier pont est distant de $600 \mu$ de la papille génitale. Le canal gynécophore est long au total de $1.250 \mu$ (après montage au baume) ou $1.450 \mu$ (exemplaire en formol). Il présente sa plus grande profondeur près de la papille génitale ou un peu en arrière; plus en arrière, il devient de plus en plus superficiel et ses lèvres s'effacent progressivement et deviennent indistinctes. Le canal proprement dit disparaît un peu en arrière des premiers testicules, mais ses lèvres plus ou moins distinctes se prolongent encore sur une certaine distance en arrière. Les testicules sont disposés sur une seule rangée, ils sont peu nombreux et il y a de longues plages qui en sont tout à fait dépourvues. Nous avons compté 57 testicules au total entre le canal gynécophore et l'extrémité postérieure du corps. Les plus petits sont ovoïdes et leur diamètre n'est que de $30 \mu$; les plus grands, ovoïdes, atteignent environ $120 \times 80 \mu$ (moyenne environ : $60 \times 90 \mu$ ). Les derniers testicules s'arrêtent à environ $600 \mu$ de l'extrémité postérieure.

Position systématique: Cette espèce se distingue de G. adami n. sp. principalement par la longueur moindre et la structure différente du canal gynécophore (bandelettes transversales moins nombreuses), et par le nombre beaucoup plus petit des testicules. 
Ces mêmes caractères séparent également cette espèce de $G$. tantali. De G. ardeolæ, elle est bien reconnaissable par la taille, deux fois plus petite, pour une longueur plus grande du canal gynécophore; par le nombre beaucoup plus réduit des testicules (57 au lieu de 700 ), etc...

Les caractères qui la séparent des cinq espèces possédant un canal gynécophore, mais décrites hors d'Afrique, sont principalement la longueur du corps et du canal gynécophore; la structure de ce dernier, la longueur de l'œsophage, etc... (voir clé).

Hôte: Veines mésentériques du Canard nain, Nettapus auritus Bodd., en association avec G. adami n. sp.

Localité et date : Astrida (Ruanda-Urundi), le 3 janvier 1956.

Type: Au Musée du Congo à Tervuren.

\section{GIGANTOBILHARZIA PLECTROPTERI n. sp.}

Cette nouvelle espèce fut découverte dans la veine-porte d'une Oie de Gambie à Astrida (Ruanda-Urundi), le 31 octobre 1955. Elle est représentée par deux femelles (dont une complète) et trois mâles (un complet). Ces spécimens sont malheureusement un peu macérés et certains caractères sont difficiles à observer.

Mâle (fig. 5-7) : Nous en avons récolté trois spécimens complets. Ceux-ci, examinés en formol, mesuraient respectivement $8-8,2$ et $9 \mathrm{~mm}$. de long. Après coloration et montage au baume, le spécimen de $8,2 \mathrm{~mm}$. (holotype) mesure encore $7,6 \mathrm{~mm}$. Voici les caractéristiques de l'holotype, examiné en formol à $5 \%$, avant coloration et montage : ver filiforme, très légèrement aplati dorso-ventralement ; cuticule dépourvue d'épines, mais finement striées. Les ventouses buccale et ventrale manquent. La bouche s'ouvre en position légèrement ventrale. Esophage long de $800 \mu$. Le pore génital est situé à $1.000 \mu$ de la bifurcation de l'œsophage. Les deux cæca se réunissent à $400 \mu$ en arrière de la bifurcation de l'œsophage. Largeur du corps au niveau de la bifurcation de l'œsophage : $45 \mu$; au niveau de la vésicule séminale : $40 \mu$; au niveau du canal gynécophore : $55 \mu$ en vue latérale et $70 \mu$ en vue dorso-ventrale ; immédiatement en arrière du canal gynécophore : 40 à $45 \mu$. Le canal gynécophore, long de $200 \mu$ environ et très peu profond, présente une courte zone très finement épineuse, immédiatement en arrière de la papille génitale. Nous n'avons pas vu d'épines sur le cirre. Le pore génital est situé ventralement dans l'angle antérieur du canal gynécophore. Nous n'avons pas vu de bandelettes transversales sur le plancher 
du canal gynécophore. Le cæcum unique, très peu sinueux, est refoulé latéralement par les testicules.

Voyons maintenant ces mensurations sur les spécimens colorés au carmin et montés en baume. Largeur à hauteur de la bifurcation de l'œsophage : 40 à $44 \mu$; au niveau du canal gynécophore, en

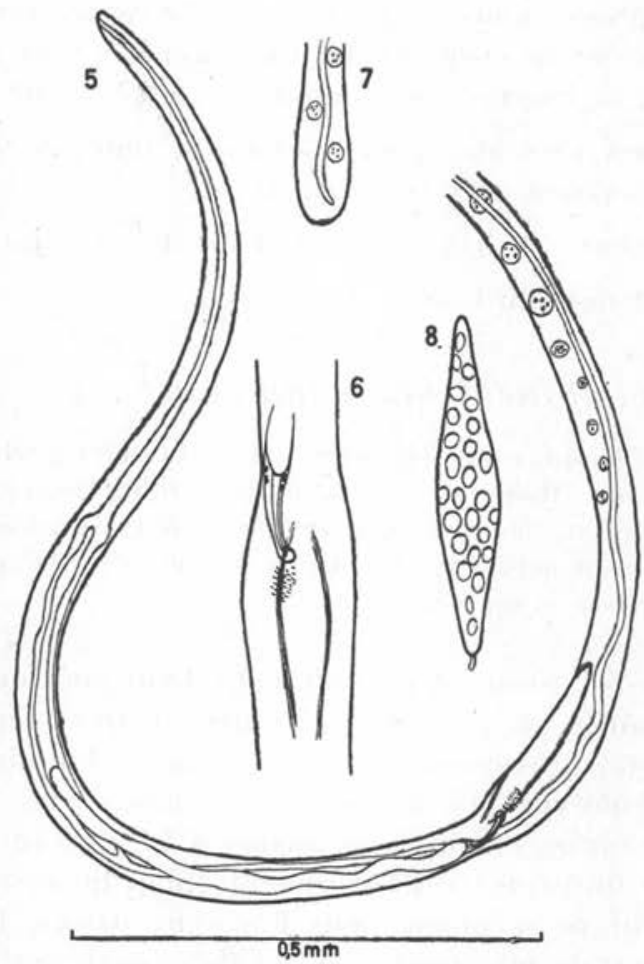

FIG. 5-8. - Gigantobilharzia plectropteri n. sp.: Mâle, partie antérieure englobant le début de la zone testiculaire (5) ; début du canal gynécophore en vue ventrale (6) ; extrémité postérieure (7). Euf dans l'ootype, chez une femelle examinée en formol (8).

vue latérale : $60 \mu$; immédiatement en arrière de ce canal : 37 à $50 \mu$; dans le tiers postérieur du corps : $42 \mu$; au niveau de l'extrémité postérieure du corps : $54 \mu$. L'extrémité postérieure du corps est légèrement dilatée et arrondie. Longueur de l'œsophage : 600 à $700 \mu$. Distance entre l'extrémité antérieure du corps et le canal gynécophore : 1.350 à $1.700 \mu$. L'union cæcale se situe à environ 
350 ou $400 \mu$ en arrière de la bifurcation de l'œsophage. Canal gynécophore long de 180 à $210 \mu$, à deux lèvres bien distinctes. Nous n'avons pas retrouvé les petites épines que nous avions observées sur les parois du canal gynécophore, immédiatement en arrière de la papille génitale. Vésicules séminales très peu distinctes, commençant un peu en avant de la jonction cæcale. Testicules peu distincts, les premiers sont visibles à environ $180 \mu$ en arrière du canal gynécophore. Ils sont disposés sur une seule rangée, leur nombre varie entre 130 et 170 environ. Les plus petits mesurent de 6 à $10 \mu$, les plus grands atteignent un diamètre de 26 à $30 \mu$. La chaîne testiculaire s'arrête à une courte distance de l'extrémité postérieure du corps.

Femelle : L'allotype, après coloration au carmin et montage en baume, mesure $6,6 \mathrm{~mm}$. de long. Le paratype incomplet est long de $6,4 \mathrm{~mm}$. (en baume); il lui manque l'extrémité antérieure en avant de la bifurcation œsophagienne et l'extrémité postérieure. Il n'y a pas de ventouse buccale, ni ventrale chez l'holotype. Cuticule comme chez le mâle. Esophage long de $675 \mu$. Distance bifurcation œsophage-extrémité antérieure de l'ootype : $450 \mu$. L'ootype est long de $100 \mu$ ( $85 \mu$ chez le paratype); son extrémité postérieure se trouve à environ $200 \mu$ de l'ovaire. Chez l'holotype, l'ootype renferme un œuf légèrement rétracté, long de $90 \mu$. Cet œuf, examiné sur le même exemplaire en formol, était allongé, légèrement fusiforme et long de $100 \mu$ pour une largeur de $18 \mu$ (fig. 8). L'ovaire est long de 350 à $400 \mu$ et décrit 18 à 22 boucles. Réceptacle séminal peu visible, mesurant $85 \mu \times 20 \mu$. Le ver présente une largeur de 45 à $52 \mu$ au niveau de la bifurcation œsophagienne. Il s'élargit légèrement en arrière et, au niveau de l'ootype, sa largeur est de 52 à $56 \mu$ environ. Immédiatement en arrière du réceptacle séminal, il est large de 65 à $70 \mu$. Au niveau de son extrémité postérieure sa largeur est de $54 \mu$. Les voies génitales ne renferment qu'un seul œuf, situé dans l'ootype. L'utérus, très long, débouche un peu en arrière de la bouche.

Position systématique : Le mâle de cette espèce est bien distinct de toutes les autres espèces décrites dans le genre Gigantobilharzia par la combinaison de plusieurs caractères, qui sont notamment le nombre relativement élevé des testicules (130 à 170), pour une longueur moyenne ou petite du corps $(7,6$ à $9 \mathrm{~mm}$.) et une brièveté exceptionnelle du canal gynécophore ( 180 à $210 \mu$ ).

La seule espèce de laquelle elle se rapproche assez étroitement est $G$. huttoni, qui est aussi une petite espèce $(4,72 \mathrm{~mm}$.), avec un 
canal gynécophore court ( 235 à $330 \mu$ ). Chez cette espèce, cependant, le nombre de testicules est beaucoup moins élevé (60); le corps est sensiblement moins large (presque 2 fois) dans les deux sexes; l'œsophage est beaucoup plus court chez la femelle : 276 à $386 \mu$ chez G. huttoni pour $675 \mu$ chez G. plectropteri (sa longueur chez le mâle n'étant pas connue pour $G$. huttoni) ; enfin, l'œuf est ovalaire chez G. huttoni, alors qu'il est fusiforme chez G. plectropteri.

Hôte: Veines portes de Plectropterus gambensis $\mathrm{L}$.

Localité et date: Astrida (Ruanda-Urundi), le 31 octobre 1955.

Types: Holotype et allotype au Musée du Congo à Tervuren.

\section{Clé du genre Gigantobilharzia (mâles seulement)}

(N. B. - Gigantobilharzia sturniæ Tanabe 1948, insuffisamment décrit, n'est pas mentionné dans cette clé).

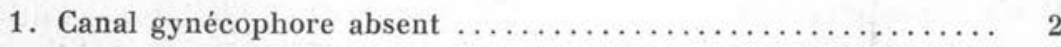

Canal gynécophore présent $\ldots \ldots \ldots \ldots \ldots \ldots \ldots \ldots \ldots \ldots \ldots \ldots \ldots \ldots \ldots \ldots \ldots \ldots \ldots$

2. Ventouse buccale présente ......... a. monocotylea Szidat 1930. Ventouse buccale absente ............... egreta Lal 1937.

3. Ventouse buccale présente, mais parfois rudimentaire ...... 4

Ventouse buccale absente ..................... 5

4. Ventouse buccale rudimentaire ; corps long de $18,5 \mathrm{~mm}$. environ, canal gynécophore long de 2,1 à 2,5 mm., avec plancher renforcé par des bandelettes finement épineuses disposées en forme d'échelle; vésicule séminale située à un millimètre de l'extrémité antérieure du corps ............ G. tantali Fain 1955.

Ventouse buccale normale ; corps plus court $(10 \mathrm{~mm})$; canal gynécophore plus court $(0,8 \mathrm{~mm}$.), sans structure épineuse scalariforme; vésicule séminale située à $0,28 \mathrm{~mm}$. de l'extrémité antérieure du corps ................. gyrauli Brackett 1940.

5. Corps très long (140 à $160 \mathrm{~mm}$.) ; canal gynécophore relativement très court $(0,55$ à $0,75 \mathrm{~mm}$.) ...... G. acotylea Odhner 1910. Corps long au maximum de $35 \mathrm{~mm}$; canal gynécophore proportionnellement beaucoup plus long. ............... 6

6. Canal gynécophore très court, ne dépassant pas $0,33 \mathrm{~mm}$. en

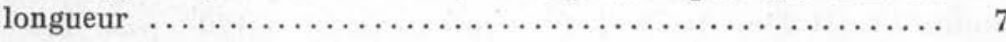

Canal gynécophore mesurant au moins $0,8 \mathrm{~mm}$. de long ..... 8

7. Corps long de $4,72 \mathrm{~mm}$. et très étroit $(0,035$ à $0,042 \mathrm{~mm}$. au niveau du canal gynécophore); canal gynécophore long de 0,235 à $0,33 \mathrm{~mm}$; 60 testicules ............. G. huttoni Leigh 1953. 
Corps plus long $(7,6 \mathrm{~mm}$. à $9 \mathrm{~mm}$.) et plus large $(0,06 \mathrm{~mm}$. au niveau du canal gynécophore) ; canal gynécophore long de 0,18 à $0,21 \mathrm{~mm}$. ; 130 à 170 testicules .......... G. plectropteri n. sp.

8. Corps très long $(35 \mathrm{~mm}$.) ; canal gynécophore proportionnellement plus court $(0,8$ à $0,9 \mathrm{~mm}$.) et renforcé par cinq bandelettes transversales; plus de 700 testicules; longueur de la vésicule séminale, 2,1 mm. ; de l'œsophage, 1,2 mm. .. G. ardeolae Fain 1956. Corps plus court (maximum $20 \mathrm{~mm}$.) ; canal gynécophore relativement plus long $(0,8$ à $2,3 \mathrm{~mm}$.), avec plancher apparemment dépourvu de bandelettes, ou avec bandelettes plus nombreuses (quatorze à quinze), ou en nombre presque égal ( $G$. nettapi), mais dans ce dernier cas les testicules sont beaucoup moins nombreux; vésicule séminale plus courte (maximum $1.095 \mathrm{~mm}$.) ; œsophage plus court (maximum 0,725 mm.) .....

9. Corps plus court $(7,3 \mathrm{~mm}$.) ; avec canal gynécophore plus long $(2,3 \mathrm{~mm}$.) ; œsophage très court $(0,3 \mathrm{~mm}$. maximum) ; vésicule séminale très courte (maximum 0,37 mm.) . G. Iaw ayi Brackett 1942.

Corps plus long (minimum $9,6 \mathrm{~mm}$. chez un jeune mâle); avec canal gynécophore plus court (maximum 1,500 mm.) : œsophage

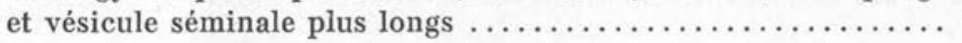

10. Canal gynécophore long de 1,250 à $1,5 \mathrm{~mm}$., avec plancher renforcé par des bandelettes cuticulaires transversales non épineuses; les deux vésicules séminales ensemble longues de 0,92 mm. au minimum ; œsophage plus long $(0,725 \mathrm{~mm}$.) ; testicules moins

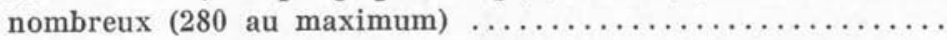

Canal gynécophore plus court $(1,084 \mathrm{~mm}$.) ; bandelettes transversales non signalées ; vésicule séminale plus courte $(0,750 \mathrm{~mm}$.) ; œsophage long au maximum de $0,69 \mathrm{~mm}$. ; 300 testicules .....

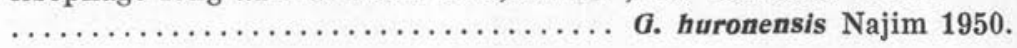

11. Corps plus court (15 mm.) mais plus large ; canal gynécophore long de $1,25 \mathrm{~mm}$., avec plancher renforcé par quatre bandelettes ; 57 testicules .............. nettapi n.

Corps plus long $(20 \mathrm{~mm}$.), mais plus étroit ; canal gynécophore long de 1,4 à $1,5 \mathrm{~mm}$., renforcé par quatorze à quinze bandelettes; 280 testicules .................. adami n sp.

\section{Bibliographie}

Brackett (S.), 1942. - Five new species of avian schistosomes from Wisconsin and Michigan with the life cycle of Gigantobilharzia gyrauli (Brack.). Journ. Parasitol., 28, 25-42.

Farv (A.), 1955a. - Recherches sur les Schistosomes d'Oiseaux au RuandaUrundi. Découverte d'une nouvelle bilharziose aviaire: la Tricho- 
bilharziose nasale et description de Schisostomes nouveaux. Note préliminaire. Rev. Zool. Bot. Afr., LI, 373-387.

FaIN (A.), 1955b. - Ettide sur les Schistosomes d'Oiseaux au Ruanda-Urundi. Un nouveau Schistosome du Tantale ibis (Ibis ibis Linn.), Gigantobilharzia tantali n. sp. Ann. Parasitol., XXX, 321-328.

FaIN (A.), 1955c. - Un nouveau Schistosome du Crabier de Madagascar (Ardeola idæe Hartl.), Gigantobilharzia ardeole n. sp. Rev. Zool. Bot. Afr., LII, 97-100.

FAIN (A.), 1956. - Les Schistosomes d'Oiseaux du genre Trichobilharzia Skrjabin et Zakharow, 1920, au Ruanda-Urundi. Rev. Zool. Bot. Afr., LIV, 147178.

LAL (M. B.), 1937. - Studies on the trematode parasites of birds. Part II. Morphology and systematic position of some blood-flukes of the family Schistosomatidæ. Journ. Parasitology, 6, (5), 274-283.

LeIGH (W. H.), 1955. - The morphology of Gigantobilharzia huttoni (Leigh, 1953) an avian schistosome with marine dermatitis-producing larvæ. Journ. Parasitol., 41, (3), 262-269.

NaJim (A. T.), 1950. — Gigantobilharzia huronensis sp. nov. a bird blood-fluke from the gold finch. Journ. Parcsit., 36, (6, Sect. 2), Suppl. p. 19.

Skruabin (R.), 1951. - Les trématodes de l'homme et des animaux. Académie des Sciences de l'U.R.S.S., Moscou, tome V, p. 397-410.

SzIDAT (L.), 1930. - Gigantobilharzia monocotylea n. sp., ein neuer Blutparasit aus Ostpreussischen Wasservogeln. Zeitschr. Parasit., 2, 583-588.

Institut de Médecine tropicale à Anvers

(Laboratoire de Zoologie médicale) 